\title{
La evaluación contínua en el Grado de Dirección y Creación de Empresa y en el Grado de Derecho de la Universidad Europea de Valencia
}

\section{Carla Paredes Gallardo a.}

a Profesora de la Universidad Europea de Valencia, España.

\begin{abstract}
Resumen
La presente comunicación tiene por objetivo presentar los resultados obtenidos de la evaluación continua desde el punto de vista de calificación final obtenida por los estudiantes comparando el efecto sobre las notas de la evaluación tradicional, basada en una prueba final única.

Se valora a un grupo de 25 estudiantes de tercero del Grado de Derecho y de Dirección y Creación de Empresas durante el segundo trimestre del curso académico 2013/2014 Y 2014/2015 en la asignatura de Derechos reales I, incluida dentro de Derecho Civil con 6 ECTS, impartida y evaluada por una sola profesora.

El trimestre está distribuido en 12 unidades didácticas, cada unidad consta de teoría, cuestiones a contestar respecto la teoría y uno o varios casos prácticos relacionados con la materia en cada una de las unidades. Al finalizar el trimestre se ha realizado un examen final.

Se trata de poner de relieve que la evaluación continua valora mejor el trabajo del estudiante durante todo el trimestre de manera continua que el examen final como prueba única para calificar el esfuerzo del estudiante.
\end{abstract}

Palabras clave: método, evaluación continua, proceso, nota final.

\section{Introducción}

Con el cambio metodológico la Universidad Europea de Valencia pretende reforzar el aprendizaje de los estudiantes de manera flexible y entretenida, fomentando la participación del estudiante en el aula, por ello considera que la evaluación continua es buena para los estudiantes ya que valora más el esfuerzo diario que la simple prueba de conocimiento al final del trimestre para evaluar el conocimiento de la materia. 
La evaluación contínua en el Grado de Dirección y Creación de Empresa y en el Grado de Derecho de la Universidad Europea de Valencia

La evaluación continua es un método de evaluación en el que se realizan pruebas periódicamente a lo largo del curso académico para que se pueda valorar el proceso de aprendizaje del estudiante y mejorarlo a medida que transcurre el curso siendo el estudiante el protagonista de su propio aprendizaje. La evaluación continua, por tanto, se inicia con una evaluación inicial, continúa a lo largo de todo el proceso y concluye con una evaluación final. La evaluación inicial nos permite conocer la situación real del estudiante en el momento del inicio del proceso, mientras que la evaluación final nos informa sobre la situación del estudiante cuando damos por concluido o cerrado el proceso. Con esto la evaluación continua nos permite conocer la situación real en cualquier momento del proceso, así como el nivel de consecución de los objetivos propuestos en cada fase.

\section{Objetivos}

Los principales objetivos del presente trabajo han sido cuatro:

En primer lugar, analizar de manera minuciosa los resultados obtenidos durante el curso académico 2013/2014 y 2014/2015 por 25 estudiantes en la asignatura de Derechos Reales I con 6 ECTS, del tercer curso del Grado de Dirección y Creación de Empresa y del Grado de Derecho de la Facultad de Ciencias Sociales de la Universidad Europea de Valencia. Dicho análisis ha consistido por un lado en evaluar las actividades de cada una de las 12 unidades didácticas y casos prácticos incluidos en cada unidad, y por otro en puntuar el examen final efectuado en la misma asignatura, para poder reflejar la repercusión de la evaluación continua en la calificación final de la asignatura.

El segundo objetivo propuesto es motivar a los estudiantes para que trabajen todos los contenidos de la asignatura tanto la teoría como la práctica desde el inicio del curso y de forma continuada durante todo el trimestre para poder adquirir plenamente los conocimientos jurídicos.

Con todo ello se pretende ayudar a los estudiantes a desarrollar competencias trasversales, tales como la comprensión del derecho como un todo sistemático y coherente, con sensibilidad hacia temas de la realidad socioeconómica, a interpretar textos jurídicos, así como argumentar y convencer jurídicamente y ser capaz de adquirir una conciencia crítica en el análisis del ordenamiento jurídico.

Por último, se pretende poder ayudar al profesor a evaluar de forma continua a los alumnos y valorar el esfuerzo continuado realizado por los estudiantes a lo largo del trimestre. 


\section{Desarrollo}

Para la consecución de los objetivos que hemos enunciado en el apartado anterior, se ha desarrollado una metodología que pasamos a describir en este apartado.

La metodología de este trabajo consistió en un análisis estadístico de las calificaciones obtenidas, por un lado mediante la evaluación de los distintos trabajos y casos prácticos que deben ir entregado a lo largo del trimestre, y por otro lado la puntuación de la prueba de conocimiento, dejando constancia como se refleja en la calificación final del curso.

El curso se distribuye en 12 unidades didácticas, compuesta cada una de ellas:

- Por un lado un conjunto de ejercicios a resolver por cada uno de los estudiantes que suponen un cierto trabajo de búsqueda y de comprensión de la teoría correspondiente, entregando las cuestiones mediante la plataforma de la asignatura.

- Por otro, de caso práctico preparado por el profesor y que deben resolver respondiendo unas preguntas sobre el caso práctico fundamentándolo jurídicamente.

El conjunto de ejercicios y la resolución de los casos prácticos incluyen criterios de evaluación cuyo principal objetivo es ayudar al estudiante, aunque también se promueven puntuaciones y se conciencia al estudiante acerca del impacto en la evaluación de las diversas partes y aspectos de los ejercicios. Este conocimiento es útil para la preparación y el estudio de pruebas posteriores

Realizando la entrega de los ejercicios y la evaluación de los ejercicios a través de la plataforma de la asignatura. 
La evaluación contínua en el Grado de Dirección y Creación de Empresa y en el Grado de Derecho de la Universidad Europea de Valencia

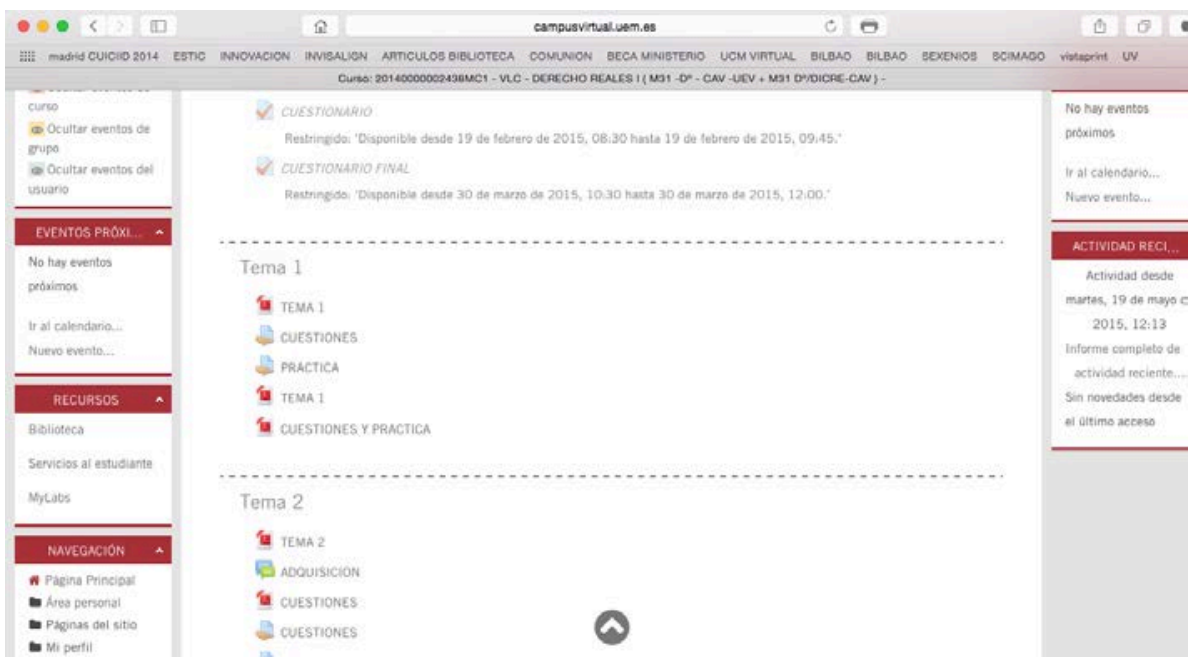

Figura 1.

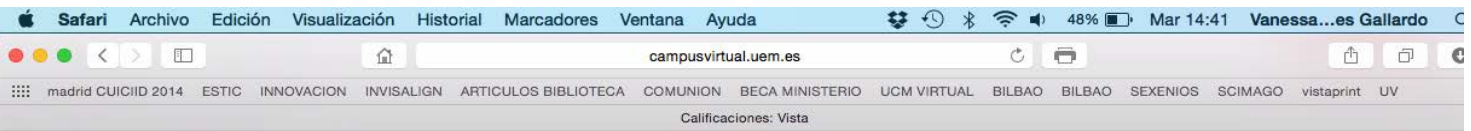

\section{Calificador}

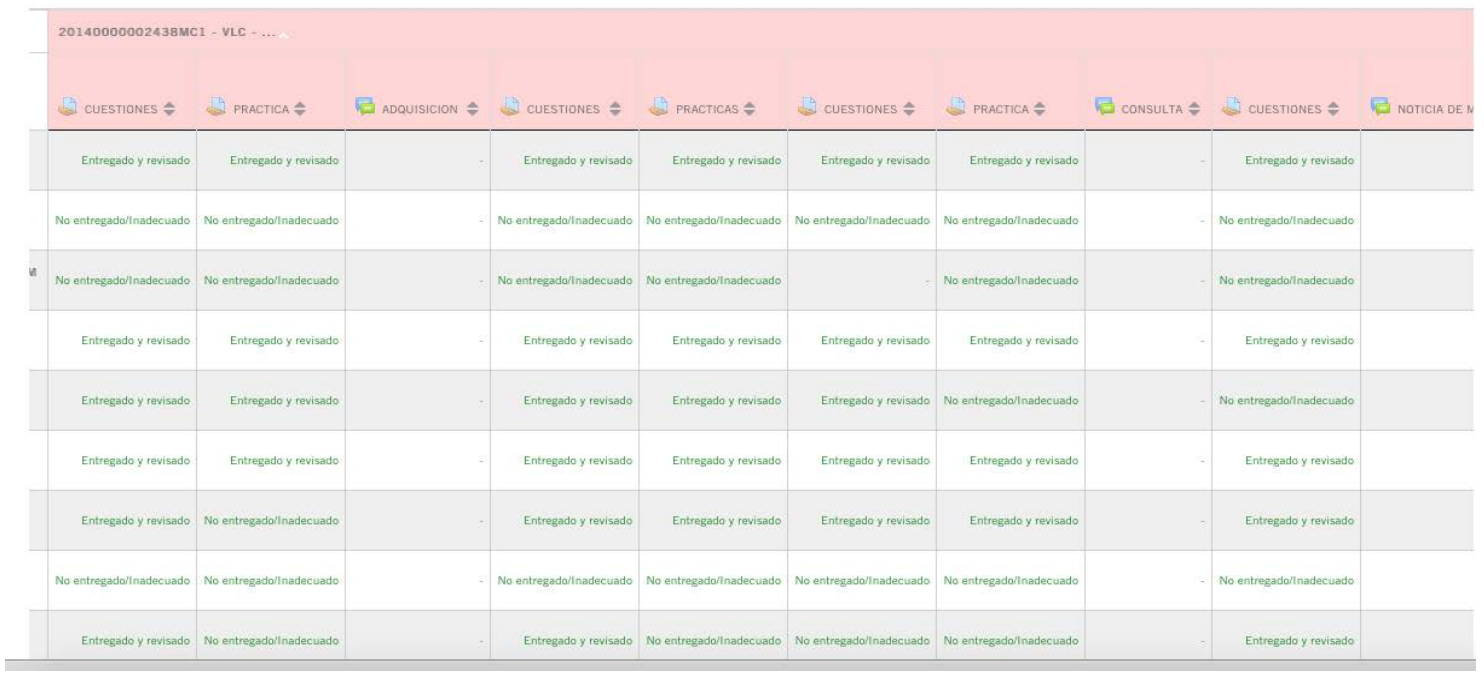

Figura 2.

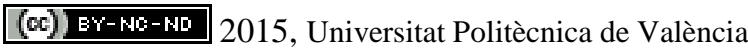


Las distintas cuestiones de las unidades didácticas y los casos prácticos se puntuaban un $50 \%$ respectivamente, y un 50\% el examen final de la asignatura, completando así la calificación final de la asignatura de Derechos Reales del cuso académico 2013/2014 y 2014/2015.

\section{Resultados}

Los resultados obtenidos en esta comunicación utilizando la metodología anteriormente expuesta muestran que la calificación final obtenida es superior a la calificación obtenida en el examen.

Se pone de manifiesto que el estudiante que trabaja de manera continua obtiene una mejor calificación final que el estudiante que solamente estudia la asignatura para poder superar el examen final, asimilando mejor los conceptos de la asignatura y teniendo una madurez jurídica mayor.

En el presente cuadro se pone de manifiesto la nota obtenida sobre 10 puntos por los alumnos en la prueba de conocimiento comparándola con la nota final obtenida después de efectuar la evaluación continua valorando la nota de la prueba de conocimiento con un 50\% y los trabajos, casos y cuestiones realizadas durante el trimestre con el resto 50\% de la puntuación.

\begin{tabular}{|c|c|c|}
\hline ALUMNO & $\begin{array}{c}\text { EXAMEN } \\
\text { FINAL }\end{array}$ & EVALUACION CONTINUA \\
\hline 1 & 4.6 & 6.9 \\
\hline 2 & 0 & 0 \\
\hline 3 & 5.5 & 8.25 \\
\hline 4 & 7.9 & 7.5 \\
\hline 5 & 6.2 & 9.3 \\
\hline 6 & 6.56 & 9.84 \\
\hline 7 & 6.86 & 10.29 \\
\hline
\end{tabular}

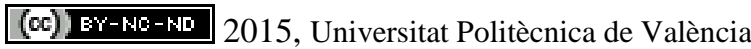


La evaluación contínua en el Grado de Dirección y Creación de Empresa y en el Grado de Derecho de la Universidad Europea de Valencia

\begin{tabular}{|c|c|c|}
\hline 8 & 0 & 0 \\
\hline 9 & 6.9 & 10.35 \\
\hline 10 & 0 & 0 \\
\hline 11 & 7.68 & 7.95 \\
\hline 12 & 5.66 & 8.49 \\
\hline 13 & 5.14 & 7.71 \\
\hline 14 & 0 & 0 \\
\hline 15 & 6.96 & 8.68 \\
\hline 16 & 7.26 & 8.83 \\
\hline 17 & 5.12 & 7.68 \\
\hline 18 & 4.6 & 6.9 \\
\hline 19 & 4.76 & 7.14 \\
\hline 20 & 5.2 & 7.8 \\
\hline 21 & NP & NP \\
\hline 22 & 1,52 & 2,28 \\
\hline 23 & 4,52 & 6,78 \\
\hline 24 & 5 & 7,5 \\
\hline 25 & 4,4 & 6,6 \\
\hline
\end{tabular}

Tabla 3.

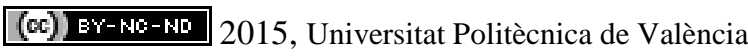




\section{Conclusión}

La aplicación de la evaluación continuada en la asignatura de Derecho Reales I ha permitido constatar una serie de ventajas e inconvenientes. En este sentido, como aspecto más favorable se ha observado que se incrementa notablemente el rendimiento académico del alumno, permitiendo una mayor distribución temporal del esfuerzo, permite el ajuste al proceso de aprendizaje gracias a una mayor interacción profesor-estudiante aumentando la implicación del estudiante y la autonomía hacia el aprendizaje.

No obstante, también se ha observado una serie de puntos débiles, como la falta de tiempo ya que son asignaturas trimestrales y la carga de trabajo por parte del estudiante y del profesor.

\section{Referencias}

CAPO PARRILLA, J., OLIVER RULLAN, X., SARD BAUZA, M. (2013) "Evaluando la evaluación continua”,@tic.revista d’innovació educativa,, nº 10, págs. 33-43.

DELGADO A.M Y OLIVER R. (2006)” La evaluación continua en el nuevo escenario docente”, Revista Universidad y Sociedad del Conocimiento (RUSC), Vol. 3 n$^{\circ} 1$ UOC.

GALLARDO GALLARDO, E., MONTOLIO D. (2011) “ Existe relación entre la evaluación continua y los resultados de los alumnos?, Revista electrónica sobre la enseñanza pública: e-publica, $n^{\circ}$ 8. págs. 63-79.

CHACON MOSCOSO, S. (2006), "Evaluación de la formación continua: fundamentos teóricos y herramientas metodológicas”, Universidad de Sevilla.

QUEROL ARAGON, E., PEREZ BENEDITO, J.L., GARCIA MARTINEZ. M.J, SEGARRA CATASUS P., (2011) "Gestión eficiente de la evaluación continua del alumnado”, Arbor: Ciencia, pensamiento y cultura, n Extra 3. Págs. 201-206.

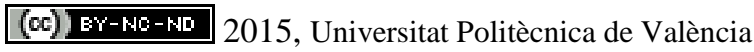

Congreso In-Red (2015) 